\title{
The Moving Carotid Artery: A Retrospective Review of the Retropharyngeal Carotid Artery and the Incidence of Positional Changes on Serial Studies
}

\author{
D.E. Lukins, S. Pilati, and E.J. Escott
}

\begin{abstract}
BACKGROUND AND PURPOSE: Retropharyngeal carotid arteries are a clinically relevant anatomic variant. Prior studies have documented their incidence, but only a single case report has discussed the change in position of the carotid artery to and from a retropharyngeal location. The purpose of this study was to determine the prevalence of retropharyngeal carotid arteries and to evaluate the change in position of retropharyngeal carotid arteries over serial CT examinations of the neck.
\end{abstract}

MATERIALS AND METHODS: A retrospective review of 306 CT examinations of the neck in 144 patients was performed. Patients with previous neck surgery or neck masses displacing the carotid arteries were excluded. The position of each carotid artery was evaluated on each examination. In patients with prior examinations, change or lack of change in position was recorded. The data were reviewed to assess changes in the position of the carotid arteries.

RESULTS: Of the 144 patients evaluated, 34 were excluded. The final number of examinations included in the study was 249 . Sixty-three of 110 patients had at least 1 comparison study. Twenty-three retropharyngeal carotid arteries were present on the baseline examination in 17 (15.5\%) of 110 patients. There was documented change to or from a retropharyngeal position in 4 (6.3\%) of 63 patients with comparison studies.

CONCLUSIONS: The phenomenon of migration of the carotid arteries to and from a retropharyngeal position with time is confirmed by our study. It is important for physicians to be aware of this phenomenon to avoid potential procedural complications.

$\mathbf{R}$ etropharyngeal carotid arteries are a well-known anatomic variant in the neck. Descriptions of this phenomenon date back to at least $1925 .{ }^{1}$ Clinical implications of this anatomic variant, including potential procedural complications, are also described in the literature of the early 20 th century ${ }^{2}$ and in more recent publications. ${ }^{3-7}$ Complications include potentially lethal hemorrhage from injury to the internal carotid artery during surgical procedures involving the pharynx, including tonsillectomy, peritonsillar abscess drainage, and transoral tumor resection. Ligation of the ICA to control hemorrhage may result in hemiplegia. ${ }^{8}$ During a transoral approach for blocking of the glossopharyngeal nerve, there may be inadvertent arterial puncture or

Received March 25, 2015; accepted after revision July 7.

From the Departments of Radiology (D.E.L. E.J.E.) and Otolaryngology Head and Neck Surgery (E.J.E.), University of Kentucky, Lexington, Kentucky; and Department of Radiology (S.P.), John H. Stroger Jr Hospital of Cook County, Chicago, Illinois.

Paper previously presented at: American Society of Neuroradiology Annual Meeting and the Foundation of the ASNR Symposium, May 17-22, 2014; Montreal, Quebec, Canada.

Please address correspondence to Douglas E. Lukins, MD, University of Kentucky, 800 Rose St, HX-302, Lexington, KY 40536; e-mail: douglas.lukins@uky.edu

http://dx.doi.org/10.3174/ajnr.A4533 injection of local anesthetic into the retropharyngeal ICA. ${ }^{3}$ Additionally, there is a risk of injury to and resultant hemorrhage from the retropharyngeal ICA during tracheal intubation. ${ }^{3}$ The retropharyngeal carotid artery has also been implicated as a potential contributing factor to obstructive sleep apnea, given its alteration of pharyngeal anatomy. ${ }^{3}$

Various theories have been proposed regarding the cause of retropharyngeal carotid arteries. Congenital alterations of the normal anatomy and increasing tortuosity of the arteries with age have both been suggested as possible causes. ${ }^{6}$ In addition to increasing age, atherosclerosis and hypertension have also been linked with abnormalities of the carotid arteries, including tortuosity, kinking, and coiling. ${ }^{9}$ Prior studies have documented the incidence of retropharyngeal carotid arteries. ${ }^{10} \mathrm{~A}$ recent study evaluated the position of the internal carotid arteries on cervical spine MR imaging. In this study, retropharyngeal carotid arteries (defined by these authors as medial to the uncovertebral joint) were present in $2.6 \%$ of patients. ${ }^{10}$ To date, only a single case report $^{11}$ (from the otolaryngology literature) has discussed the change in position of the carotid artery to and from a retropharyngeal location at 2 time points. In our own daily practice, we have noticed this change in position, which, in our experience, 

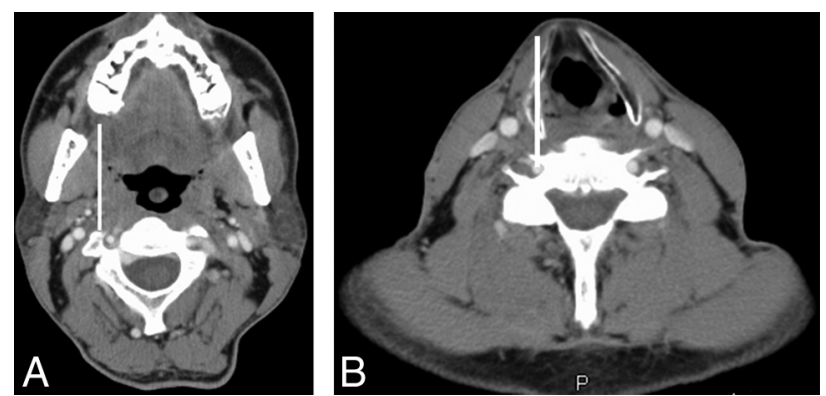

FIG 1. Reference for grading the position of the carotid artery: axial contrast-enhanced CT images of the neck soft tissues at the level of the oropharynx $(A)$ and hypopharynx $(B)$. The vertical reference lines represent the lateral margin of the pharynx. The lines are drawn along the lateral margin of the oropharyngeal wall/palatine tonsil in $A$ and the inner cortex of the thyroid cartilage (which approximates the lateral hypopharyngeal wall) in $B$. The position of the carotid artery at each level can then be graded with respect to these reference lines.

seems to occur at the level of the oropharynx and hypopharynx. The purpose of this study was to evaluate the incidence of retropharyngeal carotid arteries and their change in position over serial CT examinations of the neck.

For the purposes of this article, any reference to the carotid artery collectively refers to the common carotid artery, external carotid artery (proximal to the occipital artery), and cervical internal carotid artery.

\section{MATERIALS AND METHODS \\ Patient Demographics}

Approval was obtained from the University of Kentucky institutional review board. Contrast-enhanced CT examinations of the neck soft tissues obtained during 2012 were retrospectively evaluated in 144 patients. Patient medical record number, age, sex, and diagnosis were recorded in a spreadsheet (Excel; Microsoft, Redmond, Washington). Patients with a history of more than local neck surgery, such as extensive neck dissection that was considered to cause architectural distortion in the areas of interest, laryngectomy, or thyroidectomy, were excluded from the study. Patients with neck masses displacing the carotid arteries were also excluded, as were patients with scans that were severely degraded by motion artifacts.

\section{Image Analysis}

The examinations were all reviewed by a subspecialty board-certified neuroradiologist with or without a research assistant. The date of each initial CT examination was recorded, and the position of the carotid artery was evaluated. The right and left carotid arteries were evaluated separately. The position of the carotid artery was graded by using a scale of $0-3$, with 0 indicating a lateral position with respect to the pharynx (lateral to the line in Fig 1); 1 , a marginally retropharyngeal position (contacting the line); 2 a completely retropharyngeal position (medial to the line); and 3, a midline position (Fig 1). For carotid arteries that were given grade 1 or higher, the segment of the artery that was graded the highest (ie, positioned most toward a midline retropharyngeal location) was recorded (common carotid artery, ICA, external carotid artery). Additionally, the level of the pharynx at which the carotid artery was retropharyngeal was recorded. Finally, the prevalence of retropharyngeal carotid arteries was tabulated. The position of the ICAs at the level of the nasopharynx was not included because, in our experience, there has been no observed change in position of the ICA at this level and the fairly high frequency of vascular loops graded 2 at the level of the nasopharynx mask changes in carotid artery position at the level of the oropharynx and hypopharynx due to our method of recording only the segment with the highest grade. The goal of the study was to document changes in the position of the carotid arteries at the level of the oropharynx and hypopharynx on serial studies; therefore, our effort was focused on these levels.

The carotid arteries (of each patient) were graded with regard to the presence and extent of vessel involvement by atherosclerotic disease. Atherosclerotic plaque volume was subjectively graded on a scale of $0-3$, with 0 indicating no visible plaque; 1 , mild; 2 , moderate; and 3 , severe plaque volume, and this number was recorded on the spreadsheet. It was not feasible to calculate the degree of stenosis in the carotid arteries because of the venous phase of enhancement on most the examinations. Additionally, patients were placed into 3 categories based on the position of the carotid arteries on the baseline examination: those with at least 1 retropharyngeal carotid artery, those with at least 1 marginal carotid artery, and those with no marginal or retropharyngeal carotid artery. The average grade of atherosclerosis was calculated for each category. These data were used to evaluate any correlation between the degree of atherosclerosis and carotid artery position.

The electronic medical record was reviewed for each patient for the presence or absence of a diagnosis of hypertension on the patient's problem list. Two separate electronic medical record systems were reviewed, 1 with inpatient data and the other with outpatient data. This information was recorded on the spreadsheet.

The position of the patient's head was recorded for each CT examination. Head position was tracked on each examination by numerically grading the head position in each of 3 planes. These included "nod" or up-and-down motion of the head, "turn" or left-and-right rotation of the head about the axis of the spine, and "tilt" or left-and-right angulation of the head relative to an anteroposterior axis (Fig 2). Rotation (in each of the 3 planes) of $0^{\circ}-9^{\circ}$ was given a score of 0 , rotation of $10^{\circ}-19^{\circ}$ was given a score of 1 , and rotation of $20^{\circ}-29^{\circ}$ was given a score of 2 , and so on. Direction was indicated by "L/R" for left or right or "U/D," up or down.

After the initial CT examination was reviewed, prior examinations (up to 4) were reviewed as well. The carotid artery position was compared and recorded as either the same or different from the initial examination. If different, the exact position was graded and recorded. Additionally, head position was graded and recorded for all comparison examinations.

\section{Statistical Analysis}

Data were analyzed for correlation between age and carotid artery position, degree of atherosclerosis and carotid artery position, and diagnosis of hypertension and carotid artery position by using statistical software (JMP Pro 11.1.1; SAS Institute, Cary, North Carolina). The grades for left-and-right carotid artery po- 

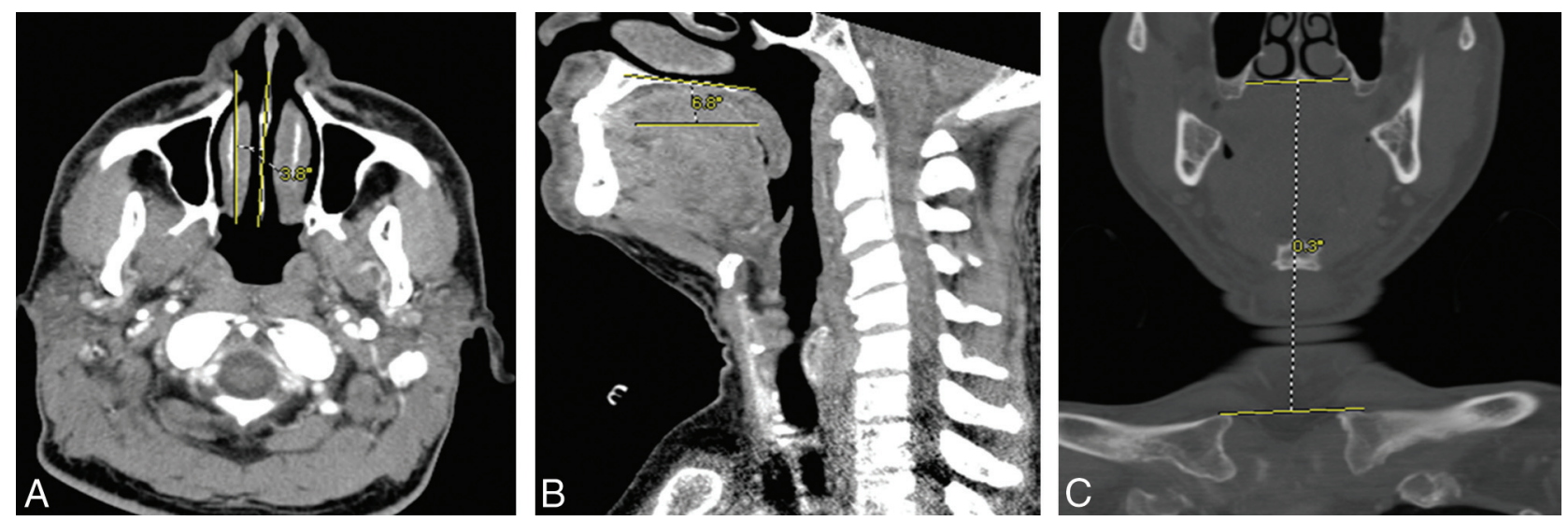

FIG 2. Images representing the "turn" $(A)$, "nod" $(B)$, and "tilt" $(C)$ axes used to calculate head position. $A$, The amount of rotation in the "turn" axis was measured in degrees of rotation of the nasal septum with respect to the vertical. $B$, The amount of rotation in the "nod" axis was measured in degrees of rotation of the hard palate from the horizontal. $C$, The amount of rotation in the "tilt" axis was measured in degrees of rotation of the hard palate with respect to a line drawn along the superior aspects of the clavicular heads.

Table 1: Patient characteristics and carotid position

\begin{tabular}{lccc}
\hline & $\begin{array}{c}\text { Only } \\
\text { Lateral }\end{array}$ & $\begin{array}{c}\text { At Least 1 Marginal and } \\
\text { No Retropharyngeal }\end{array}$ & $\begin{array}{c}\text { At Least 1 } \\
\text { Retropharyngeal }\end{array}$ \\
\hline Age (yr) (mean) (min-max) & $44.9(4-74)$ & $55.5(2-76)$ & $60.5(37-78)$ \\
$\begin{array}{c}\text { Atherosclerosis grade (mean) } \\
\text { (min-max) }\end{array}$ & $0.55(0-3)$ & $1.00(0-3)$ & $1.12(0-3)$
\end{tabular}

Note:-min indicates minimum; max, maximum.

sitions in each patient were averaged to create a single grade for each patient, and an ordinal logistic regression was performed between age and average carotid artery position. A Fisher exact test was performed to assess the correlation between the degree of atherosclerosis and carotid artery position as well as diagnosis of hypertension and carotid artery position.

\section{RESULTS}

Thirty-four of 144 patients were excluded, leaving 110 patients in the study. A prior operation was the most common reason for exclusion from the study. Of the 34 patients excluded, 24 were because of a prior neck operation. Two patients were excluded because of neck masses or lymph nodes that displaced 1 of the carotid arteries. Four patients were excluded for lack of IV contrast that limited assessment of the carotid artery position. Two patients were excluded for extensive soft-tissue emphysema. One patient was excluded for ICA occlusion; and 1 patient, for motion artifacts. Of the 110 patients included in the study, the average patient age was 50.4 years, with a range of 2-82 years. Nine patients (8\%) were in the pediatric population (younger than 18 years of age). There were 72 males and 38 females, constituting $65 \%$ and $35 \%$ of the study population, respectively.

Most patients, 74 composing $75.0 \%$ of the population, had a diagnosis of cancer. Among these patients, cancer of the aerodigestive tract was the most common diagnosis $(29.9 \%)$, followed by hematologic malignancies such as lymphoma and leukemia $(16.7 \%)$. Less common malignancies in the study population included sinonasal cancer, salivary gland cancer, endocrine gland cancer, and skin cancer. The remaining patients with diagnoses other than malignancy $(36,25.0 \%)$ were placed in an "other" category. These patients were imaged for reasons such as neck swelling, airway stenosis, sialoadenitis, abscess, and primary hyperparathyroidism.
Among the patients included in the study, the average grade of atherosclerosis was 0.76 on a scale of $0-3$. The average grade of atherosclerosis in patients whose carotid arteries showed a change in position from lateral to retropharyngeal was 1 compared with an average grade of 0.75 in the nonchanging population. Patients in the retropharyngeal category had an average grade of atherosclerosis of 1.12. Patients in the marginal category had an average grade of atherosclerosis of 1.00. Patients without a retropharyngeal or marginal carotid artery had an average grade of atherosclerosis of 0.56 (Table 1 ). There was no statistically significant correlation $(P=.128)$ between the increasing degree of atherosclerosis and increasing average carotid artery grade; however, there was a trend toward a marginal or retropharyngeal position with increasing atherosclerosis grade.

Of the 110 patients included in the study, 63 had at least 1 prior comparison examination. Some comparison studies dated back to 2000. A total of 249 examinations were evaluated, including initial examinations and comparisons. On the anchor examinations (the initial examination that was viewed for each patient), 17 of 110 patients (15.5\%) had at least 1 retropharyngeal carotid artery. Three additional patients had retropharyngeal carotid arteries on comparison examinations, but not on the anchor examination. When we took this finding into account, 20 of 110 patients (18.2\%) had at least 1 retropharyngeal carotid artery. The average age of patients with at least 1 retropharyngeal carotid artery was 60.5 years compared with patients with at least 1 marginal carotid artery (average age, 55.5 years) and patients without a retropharyngeal or marginal carotid artery (average age, 44.9 years). The youngest patient with a retropharyngeal carotid artery was 37 years of age, and the oldest patient was 78 years. The ICA was the most common retropharyngeal segment seen on the anchor examinations in our study population (61\%). The common carotid artery was the next most common retropharyngeal segment $(25 \%)$. Of note, the external carotid artery (proximal to the occipital artery) was retropharyngeal in 2 patients; this finding 

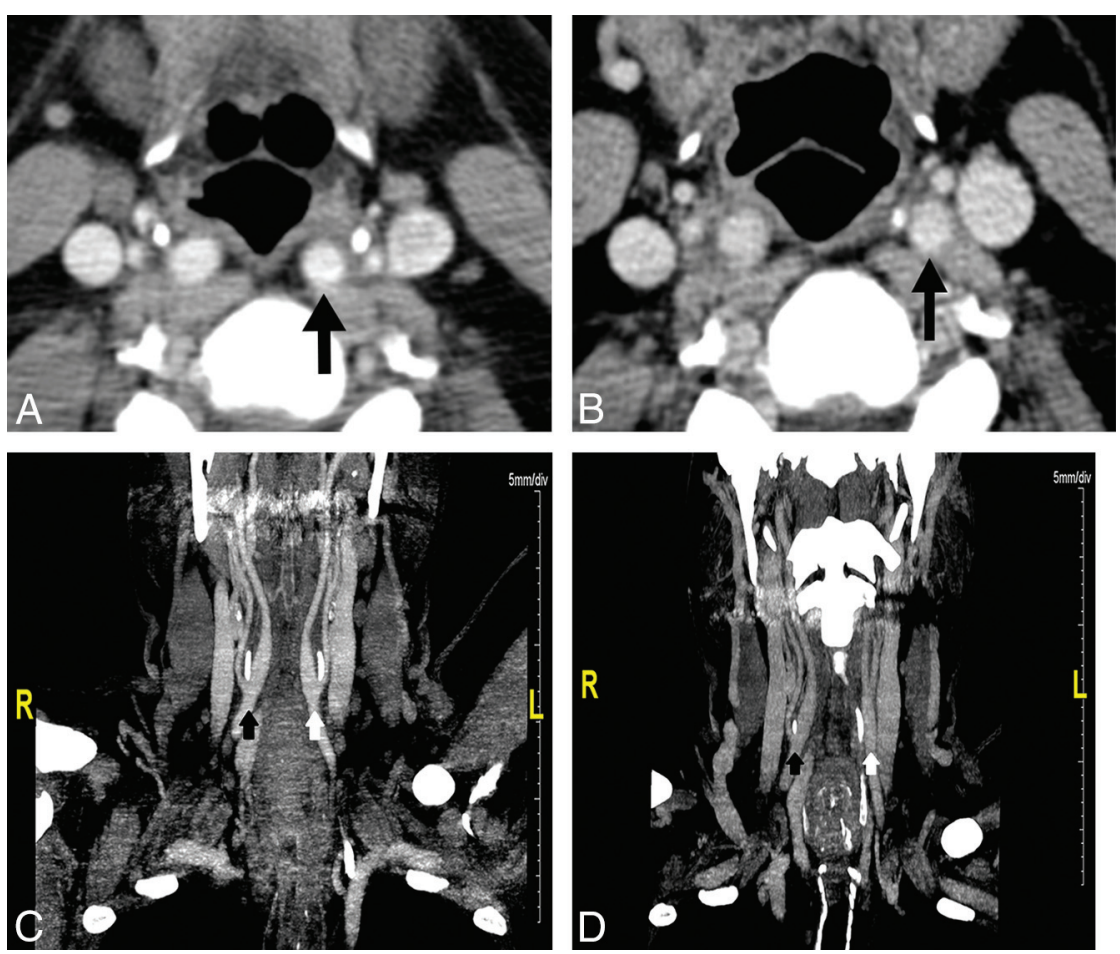

FIG 3. A 58-year-old man with mantle cell lymphoma. A, The proximal left internal carotid artery (arrow) position is retropharyngeal (grade 2). The proximal left external carotid artery can be seen lateral to the internal carotid artery. This image was obtained immediately superior to the common carotid bifurcation. The superior cornu of the thyroid cartilage lies between and just anterior to the 2 structures. $B$, On a scan obtained 1 year earlier, the proximal left internal carotid artery (arrow) position is lateral (grade 0). The proximal left external carotid artery can be seen just anterior to the internal carotid artery, and the superior cornu of the thyroid cartilage lies medial to the internal carotid artery. This image was also obtained immediately superior to the common carotid bifurcation. C, A coronal reformatted image shows the right common carotid bifurcation (black arrow) and the left common carotid bifurcation (white arrow). The superior cornu of the thyroid cartilage can be seen between the proximal internal and external carotid arteries bilaterally. Both internal carotid arteries are retropharyngeal. $D$, A coronal reformatted image from the examination obtained 1 year earlier (shown in $B$ ) shows that the proximal left internal carotid artery (white arrow) lies lateral to the superior cornu of the thyroid cartilage, whereas the proximal right internal carotid artery remains retropharyngeal.
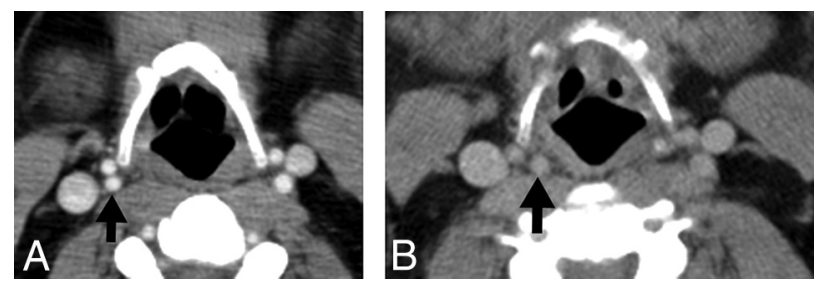

FIG 4. A 46-year-old man with Hodgkin lymphoma. $A$, The right internal carotid artery (arrow) position is lateral (grade 0). Both the proximal internal and external carotid arteries are lateral to the greater cornu of the hyoid bone. This image was obtained immediately above the level of the common carotid bifurcation. $B$, On a scan obtained 6 months earlier, the proximal right internal carotid artery (arrow) position is retropharyngeal (grade 2), located medial to the greater cornu of the hyoid bone. This image was also obtained immediately above the level of the common carotid bifurcation.

was unexpected. In addition, the carotid bifurcation was completely retropharyngeal bilaterally in 1 patient.

Logistic regression showed a statistically significant $(P<.001)$ correlation between increasing age and increasing average carotid artery grade. For example, the youngest patient included in our study (2 years of age) would have a $92.7 \%$ chance of having no retropharyngeal carotid artery (average grade, 0 ) and a $0.5 \%$ chance of having bilateral retropharyngeal carotid arteries (average grade, 2), whereas the oldest patient included in our study ( 82 years) would have a $27.0 \%$ chance of having no retropharyngeal carotid artery and a $15.4 \%$ chance of having bilateral retropharyngeal carotid arteries.

Twenty-two patients had a diagnosis of hypertension, and 88 patients did not have a diagnosis of hypertension in the electronic medical record systems. The average age of patients with a diagnosis of hypertension was 60.3 years, and the average age of those without a diagnosis of hypertension was 48.6 years. The average carotid position (on the scale of $0-3$ ) in patients with a diagnosis of hypertension was 0.568 , and the average carotid position in those without a diagnosis of hypertension was 0.381 . Of the 22 patients with a diagnosis of hypertension, $5(22.7 \%)$ had at least 1 retropharyngeal carotid artery. Of the 88 patients without a diagnosis of hypertension, 12 (13.6\%) had at least 1 retropharyngeal carotid artery. There was no statistically significant correlation $(P=.334)$ between a diagnosis of hypertension and increasing average carotid artery grade.

Within the group of 63 patients with comparison examinations, 4 patients $(6.3 \%)$ showed interval change in the position of a carotid artery from lateral to retropharyngeal or vice-versa between examinations (Figs 3 and 4). Of these 4 patients with interval change in position of the carotid arteries, the change in position occurred at the level of the oropharynx in 3 patients and at the level of the hypopharynx in 1 patient. The carotid artery that changed position was on the right in 2 patients and on the left in the other 2 patients. The segment involved was the ICA in 3 patients and the common carotid artery in the single remaining patient. The imaging features and demographic and clinical data of the 4 patients with a change in position of the carotid arteries between examinations are shown in Table 2.

Two patients whose carotid arteries changed position from lateral to retropharyngeal or vice-versa between examinations showed no change in head position between the scans when the change in position of the carotid artery occurred. The other 2 patients whose carotid artery position changed from lateral to retropharyngeal or vice-versa showed a change in the "nod" axis from $0^{\circ}$ to $10^{\circ}-19^{\circ}$ down. One of these patients had a retropharyngeal carotid artery when the head was in the $10^{\circ}-19^{\circ}$ down position and a lateral carotid artery when the head was in the neutral position. The other patient showed the opposite pattern, in which the carotid artery was retropharyngeal in the neutral position and lateral with the head in the $10^{\circ}-19^{\circ}$ down position. 
Table 2: Demographic data, diagnoses, and imaging features of patients with moving carotid arteries

\begin{tabular}{|c|c|c|c|c|c|c|c|c|}
\hline Patient & $\begin{array}{l}\text { Age } \\
\text { (yr) }\end{array}$ & Sex & Diagnosis & $\begin{array}{c}\text { Atherosclerosis } \\
\text { Grade }\end{array}$ & $\begin{array}{l}\text { Moving } \\
\text { Segment }\end{array}$ & $\begin{array}{l}\text { Level of } \\
\text { Retropharyngeal } \\
\text { Carotid }\end{array}$ & $\begin{array}{c}\text { Time } \\
\text { Interval } \\
\text { between } \\
\text { Scans (mo) }\end{array}$ & $\begin{array}{l}\text { Head } \\
\text { Position }\end{array}$ \\
\hline 1 & 58 & Male & HematoCA & 0 & Left ICA & Oropharynx & 8 & $\begin{array}{l}\text { Tilted } 10^{\circ} \text { down } \\
\text { between scans }\end{array}$ \\
\hline 2 & 46 & Male & HematoCA & 0 & Right ICA & Oropharynx & 6 & Neutral; no change \\
\hline 3 & 71 & Male & Skin cancer & 2 & Left CCA & $\begin{array}{l}\text { Hypopharynx and } \\
\text { oropharynx }\end{array}$ & 4 & $\begin{array}{l}\text { Tilted } 10^{\circ} \text { down } \\
\text { between scans }\end{array}$ \\
\hline 4 & 56 & Female & Lung cancer & 2 & Right ICA & Oropharynx & 2 & Neutral; no change \\
\hline
\end{tabular}

Note:-CCA indicates common carotid artery; HematoCA, hematologic malignancy.

In contrast, another patient changed head position from $30^{\circ}-39^{\circ}$ up to $20^{\circ}-29^{\circ}$ down between 2 scans and showed no change in position of the carotid arteries.

\section{DISCUSSION}

We showed an incidence of retropharyngeal carotid arteries on the anchor studies of $15.5 \%$; and $18.2 \%$ of patients had at least 1 retropharyngeal carotid artery found over the course of their studies. Prior studies have shown a prevalence of retropharyngeal carotid arteries of $2.6 \% .{ }^{10}$ The higher prevalence of retropharyngeal carotid arteries between our study and other studies may be related to differences in the method of determining retropharyngeal position and differences in patient demographics. Additionally, some prior studies have only commented on the retropharyngeal position of the internal carotid artery, while our study has shown that the common carotid artery and external carotid artery may also have a retropharyngeal position. Because we have demonstrated that the retropharyngeal carotid artery is not a static phenomenon, prior studies that only documented its presence at a single time point may have underestimated the true prevalence.

The average age of patients with retropharyngeal carotid arteries (60.5 years) was greater compared with those with marginal (55.5 years) or lateral (44.9 years) carotid arteries. Furthermore, statistical analysis of our data revealed a significant correlation between increasing patient age and the likelihood of having a retropharyngeal carotid artery. The association of increasing age with the presence of retropharyngeal or marginal carotid arteries was expected, because older patients tend to have a greater degree of tortuosity of the carotid arteries. ${ }^{12}$

Among our patient population, there was a trend toward association of higher grades of atherosclerosis with retropharyngeal carotid arteries compared with marginal or lateral carotid arteries. This finding was expected because patients with atherosclerotic disease frequently demonstrate tortuosity of the carotid arteries, ${ }^{9}$ and this tortuosity would logically predispose to deviation of the artery from its usual anatomic position. However, statistical analysis of our data failed to confirm that a true correlation was present. Perhaps if a larger sample size were available, a correlation would have been found, and this could potentially be a focus of further investigation.

Our data did not show a statistically significant correlation between a diagnosis of hypertension and an increasing likelihood of having a retropharyngeal carotid artery. This may have been due to incomplete charting or underreporting of hypertension in the electronic medical record, because some of the patients in our study may have been referred to our medical center for subspecialty care. In this case, the diagnosis of hypertension might have been made by their primary care provider at another institution but not entered into our electronic medical record system.

Our study demonstrated that $6.3 \%$ of patients had a change in position of the carotid arteries from retropharyngeal to lateral or vice-versa between any comparison examination and the anchor examination. To our knowledge, this incidence has never previously been evaluated. A recent case report ${ }^{11}$ documented the phenomenon of variability in the position of the retropharyngeal ICA. Variation in pharyngeal wall diameter and position of the hyoid bone with the respiratory cycle were discussed as potential explanations, but the physiology explaining this change in the position of the ICAs was thought to remain unclear. Additionally, the authors discussed whether the change in position of the retropharyngeal ICA was a fixed alteration of the anatomy of the neck or a transient variation in the position of the ICA. A recent study of the motion of parapharyngeal and retropharyngeal structures during swallowing has shown that there is anterior and medial displacement of the ICA and external carotid artery during pharyngeal contraction. ${ }^{13}$ In our study, the variability in the position of the retropharyngeal carotid arteries was at the level of the hyoid bone in all patients (Figs 2 and 3). The ICA may potentially be temporarily held in a retropharyngeal position by the tip of the greater cornu of the hyoid bone after being drawn anteromedially by pharyngeal contraction during swallowing, though this theory cannot be confirmed by our study, and the duration of the carotid artery being located in any given position cannot be known in our patient population. However, given the lack of change in carotid position in most of our patients, most carotid arteries must be relatively fixed in the retropharyngeal, marginal, or lateral positions. Much more frequent CT examinations would need to be performed on each patient to understand and fully evaluate the dynamic nature of this phenomenon.

The change in position of the head between examinations seemed to have no clear association with the change in the position of the carotid artery from lateral to retropharyngeal or viceversa. Two of the patients whose carotid arteries changed position between examinations showed no change in head position. The other 2 patients whose carotid arteries changed position showed inconsistent findings, with 1 patient demonstrating a retropharyngeal carotid artery with the head in the neutral position and the other demonstrating a retropharyngeal carotid artery with the head tilted down. Additionally, many of the patients in our study showed large changes in head position between examinations with no change in carotid artery position. 
Our patient population comprised mostly adults (92\%). This greater proportion of CT examinations performed in adults was likely due to a greater prevalence of head and neck cancer and lymphoproliferative disorders in the adult population as well as awareness of the risks of ionizing radiation to pediatric patients and resultant avoidance of CT by referring providers. While our patient population did include some pediatric patients, the results of the study apply mostly to adults because the patient population was skewed toward adults.

Most of our patient population (65\%) was male. This may potentially be explained by a higher incidence of head and neck cancer in males compared with females ${ }^{14}$ and by the large proportion of patients with cancer in our study. For this reason, however, our results may not necessarily apply to the population as a whole. Koreckij et $\mathrm{al}^{10}$ found that an aberrant course to the carotid arteries was more common in female patients and was associated with significantly greater spondylosis and kyphosis than in agematched controls.

The retropharyngeal carotid artery is a clinically important anatomic variant, and numerous complications associated with this anatomic variant have been reported in the literature. These include hemorrhage from the ICA during surgical procedures involving the pharynx, ${ }^{8}$ inadvertent puncture of and injection of anesthetic into the ICA during transoral blocking of the glossopharyngeal nerve, ${ }^{3}$ and injury of the ICA during tracheal intubation. ${ }^{3}$ Ligation of the ICA to control hemorrhage after these complications may result in hemiplegia. ${ }^{8}$ Additionally, the presence of a retropharyngeal ICA may be a contributing factor to obstructive sleep apnea. ${ }^{3}$ For these reasons, it is important to communicate the presence of a retropharyngeal carotid artery to the clinician. A carotid artery moving into and out of a retropharyngeal position further complicates matters, in that the clinician may assume that it is not present on the basis of a single imaging study and then may encounter it during an operation or in the clinic.

Our study was limited by a number of factors. The relatively small sample size may have led to overestimation or underestimation of the prevalence of retropharyngeal carotid arteries and position-changing carotid arteries. It also may have limited our ability to make a correlation between the degree of atherosclerosis and the likelihood of a retropharyngeal carotid artery. The inhomogeneous patient population in our study may also have skewed our results, though we attempted to exclude patients with conditions that would be expected to have an effect on carotid position. The inconsistent patient positioning between imaging examinations may have had an effect on carotid position, though this was unavoidable in a retrospective study and our results do not suggest that changes in patient positioning have an effect on carotid position.

\section{CONCLUSIONS}

Retropharyngeal carotid arteries are a clinically important anatomic variant, shown to occur in $18.2 \%$ of the patients in our study. Knowledge of the presence of this variant in a particular patient may allow the clinician to avoid potential complications related to surgical procedures and endotracheal intubation. Our results show that change in the position of the carotid artery from lateral to retropharyngeal or vice-versa is not an uncommon phenomenon, occurring in $6.3 \%$ of our patients. Although we did not isolate any definite causes, knowledge of this phenomenon may result in heightened awareness among physicians and therefore aid in the prevention of complications associated with this anatomic variant.

\section{REFERENCES}

1. Kelly AB. Tortuosity of the internal carotid in relation to the pharynx. J Laryngol Otol 1925;40:15-23 CrossRef

2. Skillern PG. Anomalous internal carotid artery and its clinical significance in operations on tonsils. JAMA 1913;60:172-73 CrossRef

3. Marcucci C, Thomas P, Sewell DA. Retropharyngeal carotid artery: an important anatomic variation for the anesthesiologist. Anesthesiology 2009;111:454-55 CrossRef Medline

4. Mousa AY, AbuRahma AF. Retropharyngeal internal carotid artery: a rare presentation with significant clinical implications. Ann Vasc Surg 2013:27:1189.e1-4 CrossRef Medline

5. Ozgur Z, Celik S, Govsa F, et al. A study of the course of the internal carotid artery in the parapharyngeal space and its clinical importance. Eur Arch Otorhinolaryngol 2007;264:1483-89 CrossRef Medline

6. Paulsen F, Tillman B, Christofides C, et al. Curving and looping of the internal carotid artery in relation to the pharynx: frequency, embryology and clinical implications. J Anat 2000;197:373-81 CrossRef Medline

7. Srinivasan S, Ali SZ, Chwan LT. Aberrant retropharyngeal (submucosal) internal carotid artery: an under-recognized, clinically significant variant. Surg Radiol Anat 2013;35:449-50 CrossRef Medline

8. Pfeiffer J, Ridder GJ. A clinical classification system for aberrant internal carotid arteries. Laryngoscope 2008;118:1931-36 CrossRef Medline

9. Del Corso L, Moruzzo D, Conte B, et al. Tortuosity, kinking, and coiling of the carotid artery: expression of atherosclerosis or aging? Angiology 1998;49:361-71 CrossRef Medline

10. Koreckij J, Alvi H, Gibly R, et al. Incidence and risk factors of the retropharyngeal carotid artery on cervical magnetic resonance imaging. Spine 2013;38:E109-12 CrossRef Medline

11. Gupta A, Shah AD, Zhang Z, et al. Variability in the position of the retropharyngeal internal carotid artery. Laryngoscope 2013;123: 401-03 CrossRef Medline

12. Hong JT, Kim TH, Kim IS, et al. The effect of patient age on the internal carotid artery location around the atlas. J Neurosurg Spine 2010;12:613-18 CrossRef Medline

13. Chitose S, Haraguchi M, Nagata S, et al. Analysis of passive motion of para- and retropharyngeal structures during swallowing using dynamic magnetic resonance imaging. Dysphagia 2014;29:387-95 CrossRef Medline

14. Jemal A, Bray F, Center MM, et al. Global cancer statistics. CA Cancer J Clin 2011;61:69-90 CrossRef Medline 